\title{
Safety and efficacy of carboplatin plus nab-paclitaxel for treating advanced non-small-cell lung cancer with interstitial lung disease
}

\author{
HIDEYUKI NIWA, YOSHIRO NAKAHARA, MASANORI YOKOBA, \\ HISASHI MITSUFUJI, JIICHIRO SASAKI and NORIYUKI MASUDA
}

Department of Respiratory Medicine, Kitasato University Hospital, Sagamihara, Kanagawa 252-0374, Japan

Received March 29, 2017; Accepted June 30, 2017

DOI: 10.3892/mco.2017.1359

\begin{abstract}
There are few established treatments for patients with non-small-cell lung cancer (NSCLC) with interstitial lung disease (ILD). The safety and efficacy of albumin-bound paclitaxel (nab-paclitaxel) in combination with carboplatin is uncertain, although the combination of carboplatin and paclitaxel is the most common regimen for treating NSCLC patients with ILD. A total of 9 NSCLC patients with ILD, treated between April 2013 and March 2016, were retrospectively investigated. Carboplatin (AUC 5-6) was administered on day 1 and nab-paclitaxel on days 1, 8 and 15, every 4-6 weeks. The median age of the patients upon initiating chemotherapy was 67 years. The pathological examination revealed 6 patients with squamous cell carcinoma, and 6 patients exhibited the typical pattern of ILD. The response rate was $55.6 \%$, and the median progression-free and overall survival time was 174 and 344 days, respectively. Acute exacerbation of ILD was not observed in any of the patients, and febrile neutropenia developed in 3 patients $(3 / 9,33.3 \%)$. Thus, treatment with carboplatin plus nab-paclitaxel was found to be safe and effective for NSCLC patients with ILD, although management of hematological adverse events, such as febrile neutropenia, was required. However, these encouraging results require confirmation by a large-scale clinical trial.
\end{abstract}

\section{Introduction}

There are few established treatments for patients with nonsmall-cell lung cancer (NSCLC) with interstitial lung disease (ILD). The safety and efficacy of albumin-bound paclitaxel (nab-paclitaxel) in combination with carboplatin is uncertain, although the combination of carboplatin and paclitaxel is the most common regimen for treating NSCLC patients with ILD (1-3). Lung cancer is the leading cause of cancer-related

Correspondence to: Dr Hideyuki Niwa, Department of Respiratory Medicine, Kitasato University Hospital, 1-15-1 Kitasato Minami-ku, Sagamihara, Kanagawa 252-0374, Japan

E-mail: niwahide@kitasato-u.ac.jp

Key words: non-small-cell lung cancer, interstitial lung disease, carboplatin, albumin-bound paclitaxel mortality worldwide (4), although the treatment of patients with NSCLC is gradually improving (5). For example, targeted therapies have been developed to inactivate epidermal growth factor receptor (6) and anaplastic lymphoma kinase (7). Other therapeutics include immune checkpoint inhibitors, such as nivolumab (8) and pembrolizumab (9). Clinical trials demonstrated that these treatments provided survival benefits (5). The median survival was 3.5 years for patients with an oncogenic driver and genotype-directed therapy, compared with 2.4 years for those with any oncogenic driver(s) who did not receive genotype-directed therapy. By contrast, patients with severe complications, such as ILD, are excluded from a number of clinical trials, as pre-existing ILD is a risk factor for the NSCLC therapy related to $\operatorname{ILD}(10,11)$.

ILD affects the parenchyma or the alveolar region of the lung, and it presents as an interstitial shadow on images acquired using computed tomography, according to the guidelines of American Thoracic Society and the European Respiratory Society (12). ILD, particularly idiopathic pulmonary fibrosis (IPF), is a frequent complication in patients with NSCLC (13), and the incidence of lung cancer in patients with ILD is $20-30 \%$ (11). Approximately $10 \%$ of the patients with ILD reported acute exacerbations (AE) generally characterized by suddenly progressive and severe respiratory failure, accompanied by new lung opacities and diffuse alveolar damage (14). Chemotherapy for lung cancer occasionally exacerbates AE-ILD and leads to death in $27.9 \%$ of the patients (15). In patients with advanced or recurrent NSCLC who receive at least one chemotherapy regimen, the rate of AE-ILD is higher among patients with pre-existing ILD who are administered chemotherapy, rather than among those without pre-existing ILD $(15,16)$. Pre-existing ILD was reported to be a strong risk factor in NSCLC patients, with an odds ratio of 4.80-25.27 compared with those without ILD (15).

There is no established standard regimen for NSCLC patients with ILD. The best characterized regimen for first-line chemotherapy is carboplatin (CBDCA) plus paclitaxel (PTX). However, a phase III study found that the administration of albumin-bound PTX (nab-PTX) in combination with CBDCA achieved a higher response rate compared with that of CBDCA plus PTX (33 vs. 25\%, respectively) (17). Thus, nab-PTX is a promising agent for all histological types of NSCLC. Moreover, nab-PTX may replace the conventional treatment with PTX, as it is more effective for treating patients with NSCLC. However, to the best of our knowledge, there is 
no evidence that CBDCA plus nab-PTX is safe and effective for treating patients with NSCLC with ILD. Therefore, the aim of the present retrospective study was to determine whether nab-PTX plus CBDCA is a feasible treatment option for NSCLC patients with ILD.

\section{Patients and methods}

Patient selection. A total of 9 NSCLC patients with ILD who were treated at the Kitasato University Hospital (Sagamihara, Japan) between April 2013 and March 2016 were included in this retrospective study. ILD was diagnosed according to patients' medical histories, physical examination and radiological abnormalities that were consistent with the characteristics of bilateral lung fibrosis, such as ground-glass opacity and consolidation, with or without reticular shadow. There were 34 NSCLC patients with ILD in our institution between April 2013 and March 2016. The present study included the typical interstitial pneumonia (UIP) pattern and the non-UIP pattern of ILD, according to the International Consensus Statement of the Japanese Respiratory Society (18). The inclusion criteria were as follows: i) Histologically or cytologically confirmed NSCLC, ii) clinically diagnosed pulmonary fibrosis, iii) confirmed inoperable disease, iv) age $\leq 80$ years, v) Eastern Cooperative Oncology Group (ECOG) performance status $0-2$, and vi) sufficient organ function for the administration of chemotherapy. The Institutional Ethics Review Board of Kitasato University Hospital approved the study protocol and the research was conducted in accordance with the principles of the World Medical Association Declaration of Helsinki; the patients were informed that they could withdraw consent at any time during treatment.

Treatment and methods. Patients were administered $100 \mathrm{mg} / \mathrm{m}^{2}$ nab-PTX on days 1,8 and 15 , plus CBDCA on day 1 (area under the curve=5-6) every 4-6 weeks. The suspension of nab-PTX on days 8 and 15 was permitted due to toxicities, such as hematotoxicity. In our institution, prior to the start of the treatment of this regimen and on days 8 and 15 , the patients were required to have an absolute neutrophil count of $\geq 1,000 / \mathrm{mm}^{3}$ and a platelet count of $\geq 50,000 / \mathrm{mm}^{3}$. Furthermore, granulocyte-colony stimulating factor (G-CSF) was administered for prophylaxis against hematotoxicity.

Evaluation of response. The Response Evaluation Criteria in Solid Tumors (RECIST) version 1.1 (https://ctep.cancer. gov/protocoldevelopment/docs/recist_guideline.pdf) was used to evaluate the response to treatment. Chest and abdominal $\mathrm{CT}$ was performed after every two cycles of CBDCA plus nab-PTX to evaluate tumor size.

Toxicity and acute exacerbation of ILD. Adverse events were evaluated according to the Common Terminology Criteria for Adverse Events, version 4.0 (https://evs.nci.nih. gov/ftp1/CTCAE/CTCAE_4.03_2010-06-14_QuickReference_5x7. pdf). AE-ILD was confirmed if the following criteria were met: i) Exacerbation of dyspnea within 1 month, ii) newly developed diffuse pulmonary opacity, iii) absence of heart failure and infectious lung disease, as previously described $(14,19)$. Chemotherapy-related AE-ILD was diag-
Table I. Patient characteristics $(n=9)$.

\begin{tabular}{lc}
\hline Characteristics & No. \\
\hline Sex & \\
Male/female & $9 / 0$ \\
Age, years & $67(59-76)$ \\
$\quad$ Median (range) & \\
Stage & $1 / 1 / 7$ \\
IIIA/IIIB/IV & \\
Histology & $0 / 6 / 3$ \\
AdenoCa/SCC/NOS & \\
ECOG performance status & $0 / 8 / 1$ \\
0/1/2 & \\
Smoking status & $2 / 7 / 0$ \\
Current/former/never & \\
ILD pattern & $6 / 3$ \\
UIP/non-UIP & \\
\hline
\end{tabular}

AdenoCa, adenocarcinoma; SCC, squamous cell carcinoma; NOS not otherwise specified; ECOG, Eastern Cooperative Oncology Group; ILD, interstitial lung disease; UIP, usual interstitial pneumonia.

nosed when AE-ILD developed within 28 days after the last course of chemotherapy.

Statistical analysis. The primary endpoint of the study was the incidence of AE-ILD, and the secondary endpoints were objective response rate, toxicity, median progression-free survival (PFS) and overall survival (OS). PFS and OS were analyzed using the Kaplan-Meier method. PFS was defined as the period from the first day of therapy to failure, including death and disease progression. All responses were defined according to RECIST version 1.1. OS was defined as the period from the first day of treatment to the day of death. JMP 10 software was used for all statistical analyses.

\section{Results}

Patient characteristics. The baseline patient characteristics are listed in Table I. At the time of the first administration of CBDCA plus nab-PTX, the median age of the patients was 67 years (range, 59-76 years). The ECOG performance status was $0-1$ ( $8 / 9$ patients, $88 \%)$. The disease stages were IIIA $(n=1)$, IIIB $(n=1)$ and IV $(n=7)$. The histological subtypes were squamous cell carcinoma $(n=6)$ and undifferentiated carcinoma $(n=3)$. All the patients were current or former smokers. The UIP pattern of ILD was observed in 6 patients.

Treatment delivery and response. Details of the treatment and response are shown in Table II. The median number of cycles administered was 4 (range, 1-4), 5 patients experienced a partial response, 1 patient had stable disease, and the overall response and disease control rates were 55.6 and $66.7 \%$, respectively. The median PFS was 174 days [95\% confidence interval (CI): 16-178 days; Fig. 1]. The median OS at the time of data cut-off was 355 days (95\% CI: 94-not reached; Fig. 2). 
Table II. Treatment delivery and response $(n=9)$.

\begin{tabular}{lc}
\hline Variables & No. of patients \\
\hline Treatment delivery (cycles) & 1 \\
1 & 2 \\
2 & 0 \\
3 & 6 \\
4 & \\
Response & 0 \\
Complete response & 5 \\
Partial response & 1 \\
Stable disease & 3 \\
Progressive disease & 55.6 \\
Response rate, $\%$ & 66.7 \\
Disease control rate, $\%$ & \\
\hline
\end{tabular}

Table III. Treatment-related adverse events $(n=9)$.

\begin{tabular}{lccccc}
\hline & \multicolumn{5}{c}{ Grade, $\mathrm{n}$} \\
\cline { 2 - 4 } Events & 1 & 2 & 3 & 4 & All-grade \\
\hline Leukocytopenia & 0 & 3 & 3 & 2 & 8 \\
Neutropenia & 0 & 3 & 2 & 3 & 8 \\
Thrombocytopenia & 1 & 2 & 0 & 0 & 3 \\
Anemia & 1 & 0 & 1 & 0 & 2 \\
Febrile neutropenia & - & - & 3 & 0 & 3 \\
Appetite loss & 4 & 0 & 1 & 0 & 5 \\
Constipation & 4 & 0 & 0 & 0 & 4 \\
Vomiting & 1 & 0 & 0 & 0 & 1 \\
Fatigue & 0 & 1 & 4 & 0 & 5 \\
Rash & 1 & 0 & 0 & 0 & 1 \\
Peripheral neurotoxicity & 0 & 1 & 0 & 0 & 1 \\
Bleeding & 0 & 0 & 0 & 0 & 0 \\
\hline
\end{tabular}

Toxicity and safety. The adverse events without pulmonary toxicities are shown in Table III. The most frequently reported grade $\geq 3$ hematological adverse events were leukocytopenia $(5 / 9,55.6 \%)$ and neutropenia $(5 / 9,55.6 \%)$. Febrile neutropenia (FN) occurred in $3 / 9$ patients $(33.3 \%)$, but no patient succumbed to this disorder. The most frequent adverse events were appetite loss $(5 / 9,55.6 \%)$ and fatigue $(5 / 9,55.6 \%)$.

Chemotherapy-induced AE-ILD was not observed. The 2 patients who suffered pulmonary toxicities (pulmonary infections) during CBDCA plus nab-PTX treatment were successfully treated with antibiotics.

Post-chemotherapy. After receiving CBDCA plus nab-PTX, 5 patients were administered other chemotherapies, such as S-1, docetaxel, pemetrexed and nivolumab. The details of these post-chemotherapies are presented in Table IV. One patient succumbed to AE-ILD induced by second-line pemetrexed monotherapy. A total of 5 patients succumbed to their disease during follow-up, namely 4 due to lung cancer and 1 due to AE-ILD during pemetrexed therapy.

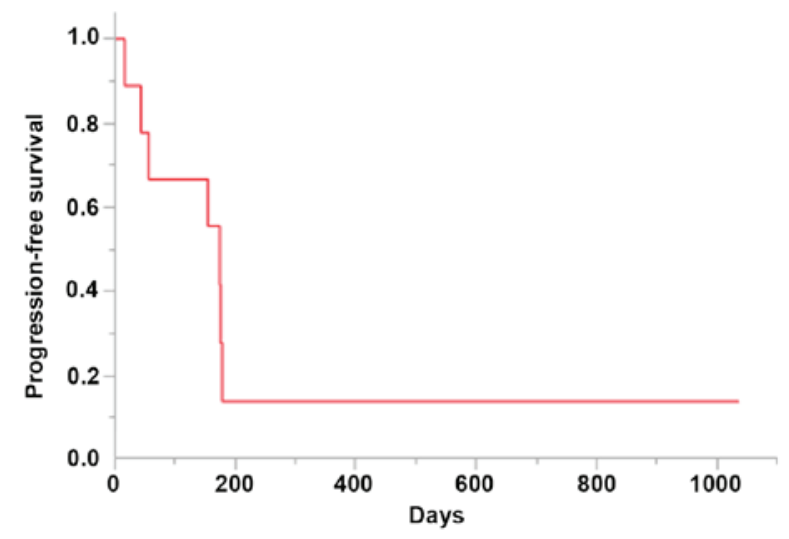

Figure 1. Kaplan-Meier analysis of the progression-free survival of the patients. Median, 174 days (5.8 months).

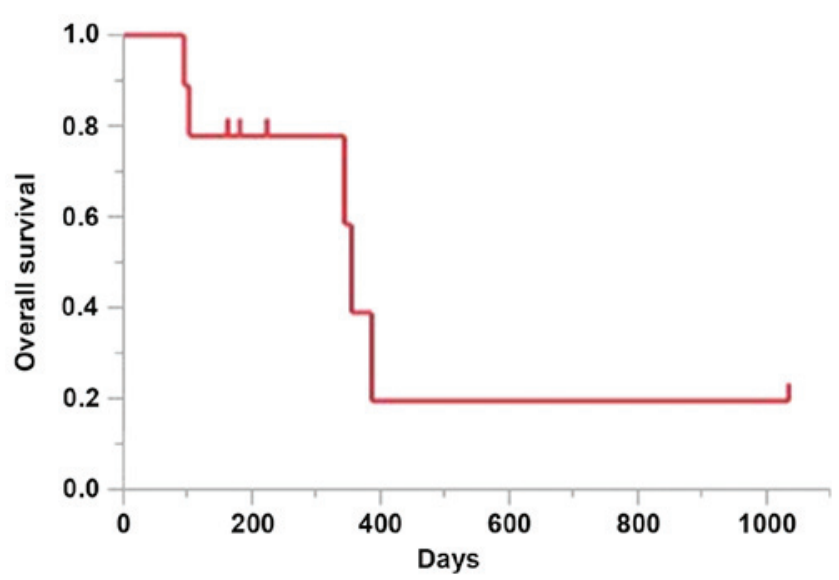

Figure 2. Kaplan-Meier analysis of the overall survival of the patients. Median, 355 days (11.8 months).

\section{Discussion}

Drug-induced AE-ILD, a potentially fatal adverse effect, may occur in NSCLC patients with ILD who undergo chemotherapy. The optimal chemotherapy for such patients remains controversial $(1,20)$, and the best-characterized regimen is CBDCA plus PTX (1-3). The efficacy of CBDCA plus nab-PTX for treating NSCLC patients without ILD has been established (17). Moreover, patients with ILD were excluded from the clinical trials (6-8). To the best of our knowledge, the present study is the first to report the efficacy and safety of CBDCA and nab-PTX in such patients.

In the present study, the overall response and disease control rates were 55.6 and $66.7 \%$, respectively, and the PFS and OS were 174 and 355 days, respectively. Previous studies analyzed the effects of CBDCA plus PTX (CP regimen) (1-3), as well as CBDCA plus PTX with bevacizumab (CPB regimen) (21,22) and CBDCA plus $\mathrm{S}-1$ (23) (Table V). The median PFS and OS of patients administered the CP regimen were 2.5-5.3 and 7.0-10.6 months, respectively (1-3) and 5.3-7.2 and 8.5-16.1 months, respectively, for those administered the CPB regimen $(21,22)$. Another study reported that the PFS and $\mathrm{OS}$ of patients treated with CBDCA plus S-1 were 4.2 and 9.7 months, respectively (23). 
Table IV. Post-chemotherapy and chemotherapy-induced AE-ILD.

\begin{tabular}{|c|c|c|c|c|c|}
\hline Patient & Age, years/sex & Histology & ILD pattern & $\operatorname{AE}-\operatorname{ILD}(+/-)$ & Post-chemotherapy AE-ILD (+/-) \\
\hline 1 & $67 / \mathrm{M}$ & $\mathrm{SCC}$ & Non-UIP & - & BSC \\
\hline 2 & $67 / \mathrm{M}$ & SCC & UIP & - & BSC \\
\hline 3 & $73 / \mathrm{M}$ & SCC & Non-UIP & - & 2nd-line S-1, 3rd-line nivolumab \\
\hline 4 & $59 / \mathrm{M}$ & Undifferentiated & UIP & - & -Pemetrexed \\
\hline 5 & 64/M & $\mathrm{SCC}$ & Non-UIP & - & $+\mathrm{S}-1$ \\
\hline 6 & 61/M & Undifferentiated & Non-UIP & - & -Docetaxel \\
\hline 7 & $66 / \mathrm{M}$ & Undifferentiated & UIP & - & $-\mathrm{BSC}$ \\
\hline 8 & $71 / \mathrm{M}$ & $\mathrm{SCC}$ & UIP & - & BSC \\
\hline 9 & $76 / \mathrm{M}$ & SCC & UIP & - & $-S-1$ \\
\hline
\end{tabular}

M, male; F, female; SCC, squamous cell carcinoma; AE, acute exacerbation; ILD, interstitial lung disease; UIP, usual interstitial pneumonia; BSC, best supportive care.

Table V. Summary of previous studies.

\begin{tabular}{|c|c|c|c|c|c|c|}
\hline Regimen & $\mathrm{N}$ & mPFS (months) & mOS (months) & AE-ILD & FN & (Refs.) \\
\hline $\mathrm{CBDCA}+\mathrm{PTX}$ & 15 & 2.5 & 7.0 & $4 / 15$ & $0 / 15$ & (20) \\
\hline CBDCA+PTX & 16 & 5.3 & 10.6 & $1 / 16$ & $1 / 16$ & (19) \\
\hline $\mathrm{CBDCA}+\mathrm{PTX}$ & 11 & 4.4 & 9.7 & $0 / 11$ & $0 / 11$ & $(21)$ \\
\hline $\mathrm{CBDCA}+\mathrm{PTX}+\mathrm{BEV}$ & 10 & 5.3 & 16.1 & $1 / 10$ & $0 / 10$ & $(21)$ \\
\hline $\mathrm{CBDCA}+\mathrm{PTX}+\mathrm{BEV}$ & 5 & ND & ND & $2 / 5$ & ND & $(18)$ \\
\hline $\mathrm{CBDCA}+\mathrm{PTX}+\mathrm{BEV}$ & 25 & 7.2 & 8.5 & $3 / 25$ & $5 / 25$ & $(22)$ \\
\hline $\mathrm{CBDCA}+\mathrm{S}-1$ & 21 & 4.2 & 9.7 & $2 / 21$ & $0 / 21$ & $(23)$ \\
\hline CBDCA+nab-PTX & 9 & 5.8 & 11.8 & $0 / 9$ & $3 / 9$ & Present \\
\hline
\end{tabular}

ND, not described; mPFS, median progression-free survival; mOS, median overall survival; AE-ILD, acute exacerbation-interstitial lung disease; FN, febrile neutropenia; CBDCA, carboplatin; PTX, paclitaxel; BEV, bevacizumab; nab-PTX, albumin-bound paclitaxel.

In the present study, chemotherapy-induced AE-ILD was not observed in patients treated with CBDCA plus nab-PTX. Previous studies demonstrated that AE-ILD occurs in 0-26.7, $10-40$ and $9.7 \%$ of patients administered the CP, CPB and CBDCA plus S-1 regimens, respectively. The present study, which was relatively small, demonstrated that treatment with CBDCA plus nab-PTX may not be the optimal option for advanced NSCLC patients with ILD.

A notable finding of the present study was that hematological adverse events were more frequent compared with those reported by other studies. The majority of the patients in our study developed leukocytopenia or neutropenia. In particular, FN occurred in 3/9 patients (Table V). Thus, the incidence of patients with FN exceeded that of patients $(<1 \%)$ without ILD in phase III clinical trials (17). Although the association between ILD and the rate of FN onset associated with this regimen was not proven, sufficient prophylaxis should be provided regardless, including administration of G-CSF, to prevent bone marrow suppression. CBDCA and nab-PTX are commonly administered triweekly (nab-PTX is administered weekly). By contrast, CBDCA and nab-PTX were administered every 4-6 weeks in the present study. The delivery period of chemotherapy was prolonged due to the frequency of hematological toxicity.
Patients without lung cancer develop acute exacerbation of ILD during the typical course of the disease. For example, a retrospective study reported that the 1-year frequency of acute exacerbation of ILD is $8.5 \%$ after diagnosis (24). Other studies reported that AE-ILD is associated with infection (25-27). Of note, we were unable to detect an association between severe hematological toxicity, including FN, in NSCLC patients with ILD, and the onset of AE-ILD.

The tyrosine kinase inhibitor nintedanib, which inhibits the receptors for vascular endothelial growth factor (VEGF), platelet-derived growth factor, and fibroblast growth factor, benefits patients with IPF (28). The administration of nintedanib is associated with a reduction in the decline of lung function, with fewer AEs and preserved quality of life (28). Furthermore, VEGF is targeted by bevacizumab and nintedanib (29). The efficacy of the prognostic extension of the $\mathrm{CPB}$ regimen for patients with non-squamous cell lung cancer is established (30). Therefore, the regimen of CBDCA plus nab-PTX with nintedanib may be an alternative useful choice for treating NSCLC patients with ILD.

There were several limitations to the present study. First, the study was retrospective and included only a limited number of patients. As mentioned above, chemotherapy for NSCLC 
patients with ILD is associated with a higher risk of AE-ILD. Thus, several of the previous studies we investigated on NSCLC patients with ILD were also small-sized. Although the clinical sample of the present study was small for oncological studies, to the best of our knowledge, ours is the first retrospective study to evaluate the efficacy and safety of the CBDCA plus nab-PTX regimen. Thus, the safety and efficacy of CBDCA plus nab-PTX must be confirmed in a prospective randomized large-scale study. Second, the follow-up time was insufficient, and only 3 patients remained alive at the time of data cut-off.

In conclusion, the present findings indicate that the CBDCA plus nab-PTX therapy regimen is an effective and safe option for patients with advanced NSCLC with pre-existing ILD, although attention must be paid to hematological toxicity. Moreover, these encouraging results require confirmation by a large-scale clinical trial.

\section{References}

1. Kenmotsu H, Naito T, Mori K, Ko R, Ono A, Wakuda K, Imai H, Taira T, Murakami H, Endo M and Takahashi T: Effect of platinum-based chemotherapy for non-small cell lung cancer patients with interstitial lung disease. Cancer Chemother Pharmacol 75: 521-526, 2015.

2. Minegishi Y, Sudoh J, Kuribayasi H, Mizutani H, Seike M, Azuma A, Yoshimura A, Kudoh S and Gemma A: The safety and efficacy of weekly paclitaxel in combination with carboplatin for advanced non-small cell lung cancer with idiopathic interstitial pneumonias. Lung Cancer 71: 70-74, 2011.

3. Shukuya T, Ishiwata T, Hara M, Muraki K, Shibayama R Koyama R and Takahashi K: Carboplatin plus weekly paclitaxel treatment in non-small cell lung cancer patients with interstitial lung disease. Anticancer Res 30: 4357-4361, 2010.

4. Siegel RL, Fedewa SA, Miller KD, Goding-Sauer A, Pinheiro PS, Martinez-Tyson D and Jemal A: Cancer statistics for Hispanics/Latinos, 2015. CA Cancer J Clin 65: 457-480, 2015.

5. Kris MG, Johnson BE, Berry LD, Kwiatkowski DJ, Iafrate AJ, Wistuba II, Varella-Garcia M, Franklin WA, Aronson SL, $\mathrm{Su} \mathrm{PF}$, et al: Using multiplexed assays of oncogenic drivers in lung cancers to select targeted drugs. JAMA 311: 1998-2006, 2014.

6. Mok TS, Wu YL, Thongprasert S, Yang CH, Chu DT, Saijo N, Sunpaweravong P, Han B, Margono B, Ichinose Y, et al: Gefitinib or carboplatin-paclitaxel in pulmonary adenocarcinoma. N Engl J Med 361: 947-957, 2009.

7. Solomon BJ, Mok T, Kim DW, Wu YL, Nakagawa K, Mekhail T, Felip E, Cappuzzo F, Paolini J, Usari T, et al: First-line crizotinib versus chemotherapy in ALK-positive lung cancer. N Engl J Med 371: 2167-2177, 2014.

8. Borghaei H, Paz-Ares L, Horn L, Spigel DR, Steins M, Ready NE, Chow LQ, Vokes EE, Felip E, Holgado E, et al: Nivolumab versus docetaxel in advanced nonsquamous non-small-cell lung cancer. N Engl J Med 373: 1627-1639, 2015.

9. Herbst RS, Baas P, Kim DW, Felip E, Pérez-Gracia JL, Han JY, Molina J, Kim JH, Arvis CD, Ahn MJ, et al: Pembrolizumab versus docetaxel for previously treated,PD-L1-positive, advanced non-small-cell lung cancer (KEYNOTE-010): A randomised controlled trial. Lancet 387: 1540-1550, 2016.

10. Isobe K, Hata Y, Sakamoto S, Takai Y, Shibuya K and Homma S: Clinical characteristics of acute respiratory deterioration in pulmonary fibrosis associated with lung cancer following anti-cancer therapy. Respirology 15: 88-92, 2010.

11. Minegishi Y, Takenaka K, Mizutani H, Sudoh J, Noro R, Okano T, Azuma A, Yoshimura A, Ando M, Tsuboi E, et al: Exacerbation of idiopathic interstitial pneumonias associated with lung cancer therapy. Intern Med 48: 665-672, 2009.

12. American Thoracic Society; European Respiratory Society: American Thoracic society/European respiratory society international multidisciplinary consensus classification of the idiopathic interstitial pneumonias. This joint statement of the American thoracic society (ATS) and the European respiratory society (ERS) was adopted by the ATS board of directors, June 2001 and by the ERS executive committee, June 2001. Am J Respir Crit Care Med 165: 277-304, 2002.
13. Ozawa Y, Suda T, Naito T, Enomoto N, Hashimoto D, Fujisawa T, Nakamura Y, Inui N, Nakamura $\mathrm{H}$ and Chida $\mathrm{K}$ : Cumulative incidence of and predictive factors for lung cancer in IPF. Respirology 14: 723-728, 2009.

14. Kondoh Y, Taniguchi H, Kawabata Y, Yokoi T, Suzuki K and Takagi K: Acute exacerbation in idiopathic pulmonary fibrosis. Analysis of clinical and pathologic findings in three cases. Chest 103: 1808-1812, 1993.

15. Kudoh S, Kato H, Nishiwaki Y, Fukuoka M, Nakata K, Ichinose Y, Tsuboi M, Yokota S, Nakagawa K, Suga M, et al: Interstitial lung disease in Japanese patients with lung cancer: A cohort and nested case-control study. Am J Respir Crit Care Med 177: 1348-1357, 2008.

16. Kenmotsu H, Naito T, Kimura M, Ono A, Shukuya T, Nakamura Y, Tsuya A, Kaira K, Murakami H, Takahashi T, et al: The risk of cytotoxic chemotherapy-related exacerbation of interstitial lung disease with lung cancer. J Thorac Oncol 6: 1242-1246, 2011

17. Socinski MA, Bondarenko I, Karaseva NA, Makhson AM, Vynnychenko I, Okamoto I, Hon JK, Hirsh V, Bhar P, Zhang H, et al: Weekly nab-paclitaxel in combination with carboplatin versus solvent-based paclitaxel plus carboplatin as first-line therapy in patients with advanced non-small-cell lung cancer: Final results of a phase III trial. J Clin Oncol 30: 2055-2062, 2012.

18. Raghu G, Rochwerg B, Zhang Y, Garcia CA, Azuma A, Behr J, Brozek JL, Collard HR, Cunningham W, Homma S, et al: An official ATS/ERS/JRS/ALAT clinical practice guideline: Treatment of idiopathic pulmonary fibrosis. An update of the 2011 clinical practice guideline. Am J Respir Crit Care Med 192: e3-e19, 2015

19. Azuma A,Nukiwa T, Tsuboi E, Suga M, Abe S, Nakata K, Taguchi Y, Nagai S, Itoh H, Ohi M, et al: Double-blind, placebo-controlled trial of pirfenidone in patients with idiopathic pulmonary fibrosis. Am J Respir Crit Care Med 171: 1040-1047, 2005.

20. Ohe Y, Ohashi Y, Kubota K, Tamura T, Nakagawa K, Negoro S, Nishiwaki Y, Saijo N, Ariyoshi Y and Fukuoka M: Randomized phase III study of cisplatin plus irinotecan versus carboplatin plus paclitaxel, cisplatin plus gemcitabine, and cisplatin plus vinorelbine for advanced non-small-cell lung cancer: Four-Arm cooperative study in Japan. Ann Oncol 18: 317-323, 2007.

21. Shimizu R, Fujimoto D, Kato R, Otoshi T, Kawamura T, Tamai K, Matsumoto T, Nagata K, Otsuka K, Nakagawa A, et al: The safety and efficacy of paclitaxel and carboplatin with or without bevacizumab for treating patients with advanced nonsquamous non-small cell lung cancer with interstitial lung disease. Cancer Chemother Pharmacol 74: 1159-1166, 2014.

22. Enomoto Y, Kenmotsu H, Watanabe N, Baba T, Murakami H, Yoh K, Ogura T, Takahashi T, Goto K and Kato T: Efficacy and safety of combined carboplatin, paclitaxel, and bevacizumab for patients with advanced non-squamous non-small cell lung cancer with pre-existing interstitial lung disease: A retrospective multi-institutional study. Anticancer Res 35: 4259-4263, 2015.

23. Sekine A, Satoh H, Baba T, Ikeda S, Okuda R, Shinohara T, Komatsu S, Hagiwara E, Iwasawa T, Ogura T and Kato T: Safety and efficacy of S-1 in combination with carboplatin in non-small cell lung cancer patients with interstitial lung disease: A pilot study. Cancer Chemother Pharmacol 77: 1245-1252, 2016.

24. Kim DS, Park JH, Park BK, Lee JS, Nicholson AG and Colby T: Acute exacerbation of idiopathic pulmonary fibrosis: Frequency and clinical features. Eur Respir J 27: 143-150, 2006.

25. Konishi K, Gibson KF, Lindell KO, Richards TJ, Zhang Y, Dhir R, Bisceglia M, Gilbert S, Yousem SA, Song JW, et al: Gene expression profiles of acute exacerbations of idiopathic pulmonary fibrosis. Am J Respir Crit Care Med 180: 167-175, 2009.

26. Wootton SC, Kim DS, Kondoh Y, Chen E, Lee JS, Song JW, Huh JW, Taniguchi H, Chiu C, Boushey H, et al: Viral infection in acute exacerbation of idiopathic pulmonary fibrosis. Am J Respir Crit Care Med 183: 698-702,2011.

27. Ushiki A, Yamazaki Y, Hama M, Yasuo M, Hanaoka M and Kubo K: Viral infections in patients with an acute exacerbation of idiopathic interstitial pneumonia. Respir Investig 52: 65-70, 2014.

28. Richeldi L, du Bois RM, Raghu G, Azuma A, Brown KK, Costabel U, Cottin V, Flaherty KR, Hansell DM, Inoue Y, et al: Efficacy and safety of nintedanib in idiopathic pulmonary fibrosis. N Engl J Med 370: 2071-2082, 2014.

29. Manzo A, Carillio G, Montanino A, Costanzo R, Sandomenico C, Rocco G and Morabito A: Focus on nintedanib in NSCLC and other tumors. Front Med (Lausanne) 3: 68, 2016.

30. Sandler A, Gray R, Perry MC, Brahmer J, Schiller JH, Dowlati A, Lilenbaum $\mathrm{R}$ and Johnson DH: Paclitaxel-carboplatin alone or with bevacizumab for non-small-cell lung cancer. N Engl J Med 355: 2542-2550, 2006. 